11

\title{
Угловое и спектральное распределения сверхширокополосного излучения фотоэмиссионного источника
}

\author{
(C) A.А Кондратьев \\ Госкорпорация «Росатом» Российский федеральный ядерный центр - \\ Всероссийский научно-исследовательский институт технической фризики им. акад. Е.И. Забабахина, \\ 456770 Снежинск, Челябинская обл., Россия \\ e-mail: dep5@vniitf.ru
}

(Поступило в Редакцию 14 ноября 2016 г. В окончательной редакции 23 августа 2017 г.)

Получена угловая зависимость напряженности электрического поля электромагнитной волны, создаваемой прямоугольным фотоэмиссионным источником с заданной поверхностной плотностью дипольного момента. Излучение диполей инициируется волной, распространяющейся вдоль поверхности со сверхсветовой фазовой скоростью. Проведено спектральное разложение излучения и вычислено угловое распределение энергии излучения.

DOI: 10.21883/JTF.2018.03.45605.2095

\section{Введение}

Теоретическая модель генерации сверхширокополосного электромагнитного излучения (ЭМИ) при наклонном облучении плоской поверхности рентгеновским излучением была предложена в работах $[1,2]$. Для увеличения мощности ЭМИ было предложено использовать вакуумный диод с анодом, прозрачным для инициирующего излучения и ускоренных электронов $[3,4]$. Результаты экспериментов по генерации сверхширокополосного ЭМИ с инициированием разряда в вакуумном анодкатодном зазоре плоской волной лазерного излучения пикосекундной длительности, падающей наклонно на фотокатод, приведены в работе [5]. Так как волна, инициирующая разряд, движется вдоль катода с фазовой скоростью, превышающей скорость света, такой источник также называют сверхсветовым. Показано [1,3], что когерентное сложение излучения электронов происходит в направлении зеркального отражения инициирующего излучения от катода, а напряженность поля в волновой зоне определяется второй производной по времени от поверхностной плотности дипольного момента.

Ширина спектра фотоэмиссионного источника ЭМИ [5] составляет 10-15 GHz. Такой источник может быть использован для сверхширокополосной радиолокации, в том числе для поиска мин и радиолокации лесных массивов, а также для исследований влияния мощного электромагнитного излучения сантиметрового диапазона на различную радиоэлектронную аппаратуру.

Для практических применений необходимо знать угловое и спектральное распределения ЭМИ. В настоящей работе для прямоугольной однородно излучающей области получены аналитические выражения для напряженности поля в волновой зоне в виде функции от поверхностной плотности дипольного момента и его производных по времени. Зависимость второй производной плотности дипольного момента от времени может быть определена с использованием аналитических моде- лей $[2,6,7]$, численными расчетами или экспериментальными измерениями напряженности поля в направлении зеркального отражения инициирующего излучения от катода. Используя полученные уравнения для напряженности поля, проведено спектральное разложение и вычислено угловое распределение энергии ЭМИ.

\section{Угловая зависимость напряженности поля излучения}

Задача решается в следующей постановке. В плоскости $z=0$ в прямоугольной области, показанной на рис. 1, находятся излучатели с поверхностной плотностью дипольного момента

$$
\begin{aligned}
& \mathbf{p}(x, y, t)= \\
& \begin{cases}\mathbf{n}_{z} p_{0} f\left(t-x \sin \vartheta_{0} / c\right), & 0 \leq x \leq a, 0 \leq y \leq b, \\
0, & \text { в остальной области, }\end{cases}
\end{aligned}
$$

где $\mathbf{n}_{z}$ - единичный вектор, направленный вдоль оси $z$, $f(t)$ - заданная функция. Зависимость плотности дипольного момента от времени вида (1) соответствует инициированию излучения диполей плоской волной, падающей на плоскость $z=0$ под уголом $\vartheta_{0} \neq 0$. В экспериментах [5] такое инициирование осуществляется лазерным излучением пикосекундной длительности, падающим на плоский фотокатод вакуумного фотодиода.

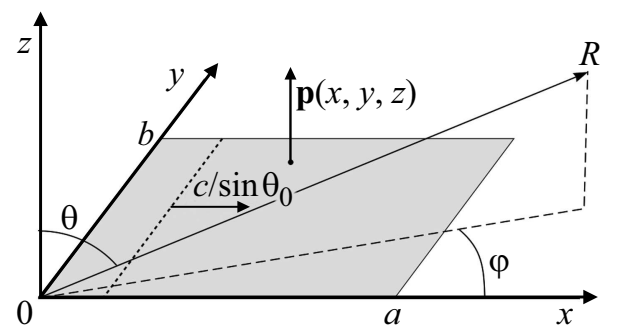

Рис. 1. Геометрия решаемой задачи. 
Обозначим $f_{1}(t)=d f / d t, f_{2}(t)=d f_{1} / d t$. В точке со сферическими координатами $\left(R_{0}, \vartheta, \varphi\right)$ на расстоянии $R_{0}$, много большем размеров излучающей системы и характерной длины волны излучения, напряженность поля определяется интегралом от второй производной плотности дипольного момента по времени с учетом запаздывания [8]

$$
\begin{aligned}
& E_{\vartheta}\left(t+R_{0} / c\right)=\frac{p_{0} \sin \vartheta}{R_{0} c^{2}} \int_{0}^{a} d x \\
& \times \int_{0}^{b} f_{2}\left(t+\frac{x}{c}\left(\sin \vartheta \cos \varphi-\sin \vartheta_{0}\right)+\frac{y}{c} \sin \vartheta \sin \varphi\right) d y .
\end{aligned}
$$

В этом уравнении использовано условие $R_{0} \gg a, b$ для вычисления расстояния от точки с координатами $(x, y, 0)$ до точки наблюдения $\left(R_{0}, \vartheta, \varphi\right)$

$$
\begin{aligned}
R(x, y) & =\left(R_{0}^{2}+x^{2}+y^{2}-2 R_{0} \sin \vartheta(x \cos \varphi+y \sin \varphi)\right)^{1 / 2} \\
& \approx R_{0}-\sin \vartheta(x \cos \varphi+y \sin \varphi) .
\end{aligned}
$$

Обозначим

$$
t_{a}=\frac{a}{c}\left(\sin \vartheta \cos \varphi-\sin \vartheta_{0}\right), t_{b}=\frac{b}{c} \sin \vartheta \sin \varphi .
$$

Разность времени прихода излучения с одинаковой фазой из начала координат и из точки с координатой $(a, 0,0)$ в точку наблюдения $\left(R_{0}, \vartheta, \varphi\right)$ равна $t_{a}$. Аналогично $t_{b}$ - разность времени прихода излучения с одинаковой фазой из начала координат и из точки с координатой $(0, b, 0)$.

Для различных $(\vartheta, \varphi)$ возможны следующие случаи. 1. $t_{a}=t_{b}=0 \quad\left(\varphi=0\right.$ и $\left.\sin \vartheta=\sin \vartheta_{0}\right)$. В этом случае излучение всех диполей складывается когерентно и уравнение (2) имеет вид

$$
E_{\vartheta}\left(t+R_{0} / c\right)=\frac{p_{0} a b \sin \vartheta_{0}}{R_{0} c^{2}} f_{2}(t) .
$$

2. $t_{a} \neq 0, t_{b}=0(\varphi=0, \pi)$. Функция $f_{2}$ в уравнении (2) не зависит от $y$.

$$
E_{\vartheta}\left(t+R_{0} / c\right)=\frac{p_{0} a b \sin \vartheta}{R_{0} c^{2}} \frac{\left[f_{1}\left(t+t_{a}\right)-f_{1}(t)\right]}{t_{a}} .
$$

3. $t_{a}=0, t_{b} \neq 0$. Функция $f_{2}$ в уравнении (2) не зависит от $x$.

$$
E_{\vartheta}\left(t+R_{0} / c\right)=\frac{p_{0} a b \sin \vartheta}{R_{0} c^{2}} \frac{\left[f_{1}\left(t+t_{b}\right)-f_{1}(t)\right]}{t_{b}} .
$$

4. $t_{a} \neq 0, t_{b} \neq 0$.

$$
\begin{aligned}
& E_{\vartheta}\left(t+R_{0} / c\right)=\frac{p_{0} a b \sin \vartheta}{R_{0} c^{2}} \\
& \times \frac{f\left(t+t_{a}+t_{b}\right)-f\left(t+t_{b}\right)-f\left(t+t_{a}\right)+f(t)}{t_{a} t_{b}} .
\end{aligned}
$$

Уравнения (4)-(6) также могут быть получены из уравнения (7) соответственно в пределе $t_{a} \rightarrow 0$ и/или $t_{b} \rightarrow 0$.

\section{Спектральное разложение излучения}

Энергия $d W$, излученная в элементе телесного угла $d \Omega$, вычисляется интегрированием вектора Пойнтинга по времени

$$
d W=d \Omega \frac{c R_{0}^{2}}{4 \pi} \int_{-\infty}^{+\infty} E^{2} d t=d \Omega \frac{c R_{0}^{2}}{4 \pi} \int_{-\infty}^{+\infty}|E(\omega)|^{2} d \omega
$$

где

$$
E(\omega)=\frac{1}{\sqrt{2 \pi}} \int_{-\infty}^{+\infty} E(t) e^{-i \omega t} d t
$$

Вычислим преобразование Фурье выражения (7)

$$
\begin{aligned}
E_{\vartheta}\left(\omega, R_{0}, \vartheta, \varphi\right)= & -\frac{p_{0} a b \sin \vartheta}{R_{0} c^{2} t_{a} t_{b}} 4 f(\omega) \\
& \times \exp \left(\frac{i \omega\left(t_{a}+t_{b}\right)}{2}\right) \sin \frac{\omega t_{a}}{2} \sin \frac{\omega t_{b}}{2},
\end{aligned}
$$

где $f(\omega)$ - преобразование Фурье функции $f(t)$, и воспользуемся соотношением $f_{2}(\omega)=-\omega^{2} f(\omega)$ :

$$
\begin{aligned}
\mid E_{\vartheta}(\omega, & \left.R_{0}, \vartheta, \varphi\right)\left.\right|^{2}=\left(\frac{p_{0} a b}{R_{0} c^{2}}\right)^{2} \sin ^{2} \vartheta\left|f_{2}(\omega)\right|^{2} \\
& \times \frac{\sin ^{2}\left(\omega t_{a} / 2\right)}{\left(\omega t_{a} / 2\right)^{2}} \frac{\sin ^{2}\left(\omega t_{b} / 2\right)}{\left(\omega t_{b} / 2\right)^{2}} .
\end{aligned}
$$

Полученное уравнение можно также записать в виде

$$
\begin{aligned}
\left|E_{\vartheta}\left(\omega, R_{0}, \vartheta, \varphi\right)\right|^{2}=\left|E_{\vartheta}\left(\omega, R_{0}, \vartheta_{0}, 0\right)\right|^{2} \\
\times \frac{\sin ^{2} \vartheta}{\sin ^{2} \vartheta_{0}} \frac{\sin ^{2}\left(\omega t_{a} / 2\right)}{\left(\omega t_{a} / 2\right)^{2}} \frac{\sin ^{2}\left(\omega_{b} / 2\right)}{\left(\omega t_{b} / 2\right)^{2}} .
\end{aligned}
$$

\section{Угловое распределение энергии излучения}

Для вычисления углового распределения энергии излучения в соответствии с (8) можно проинтегрировать $E^{2}(t)$ по времени, либо $|E(\omega)|^{2}$ по частотам. Воспользуемся уравнением (7)

$$
\begin{aligned}
\frac{d W}{d \Omega}= & \frac{\left(p_{0} a b\right)^{2} \sin ^{2} \vartheta}{4 \pi c^{3} t_{a}^{2} t_{b}^{2}} \int_{-\infty}^{+\infty}\left[f\left(t+t_{a}+t_{b}\right)\right. \\
& \left.-f\left(t+t_{a}\right)-f\left(t+t_{b}\right)+f(t)\right]^{2} d t .
\end{aligned}
$$

Обозначим $\Psi(\tau) \equiv \int_{-\infty}^{+\infty} f(t) f(t+\tau) d t$, тогда

$$
\begin{aligned}
\frac{d W}{d \Omega}= & \frac{\left(p_{0} a b\right)^{2} \sin ^{2} \vartheta}{2 \pi c^{3} t_{a}^{2} t_{b}^{2}}\left(2 \Psi(0)-2 \Psi\left(t_{a}\right)-2 \Psi\left(t_{b}\right)\right. \\
& \left.+\Psi\left(t_{a}+t_{b}\right)+\Psi\left(t_{a}-t_{b}\right)\right)
\end{aligned}
$$


Более простое выражение получается для распределения энергии излучения в плоскости $x z$ (см. уравнение (5))

$$
\frac{d W}{d \Omega}=\frac{\left(p_{0} a b\right)^{2} \sin ^{2} \vartheta}{2 \pi c^{3} t_{a}^{2}} \int_{-\infty}^{+\infty}\left(f_{1}^{2}(t)-f_{1}(t) f_{1}\left(t+t_{a}\right)\right) d t .
$$

Произведем вычисления для излучателя, у которого вторая производная дипольного момента от времени имеет вид

$$
f_{2}(t)=\omega_{0}^{2} \begin{cases}\sin \left(\omega_{0} t\right), & 0 \leq t \leq 2 \pi N / \omega_{0} \\ 0, & t<0, t>2 \pi N / \omega_{0}\end{cases}
$$

где $N$ - натуральное число. Выбор такой зависимости обусловлен тем, что она позволяет получить простые аналитические выражения и для $N=1$ дает широкий спектр излучения, а для больших $N-$ узкий. Спектральное разложение этой функции

$$
\left|f_{2}(\omega)\right|^{2}=\frac{2 \omega_{0}^{2}}{\pi} \frac{\sin ^{2}\left(\pi N \omega / \omega_{0}\right)}{\left(1-\omega^{2} / \omega_{0}^{2}\right)^{2}} .
$$

В направлении $\vartheta=\vartheta_{0}, \varphi=0$

$$
\frac{d W\left(\vartheta_{0}, 0\right)}{d \Omega}=\frac{\left(p_{0} a b\right)^{2} N \omega_{0}^{3} \sin ^{2} \vartheta_{0}}{4 c^{3}} .
$$

В плоскости $x z$ в случае $\left|\omega_{0} t_{a}\right|<2 \pi N$

$$
\frac{d W(\vartheta)}{d \Omega}=\frac{d W\left(\vartheta_{0}, 0\right)}{d \Omega} \frac{\sin ^{2} \vartheta}{\sin ^{2} \vartheta_{0}} g\left(\omega_{0} t_{a}\right),
$$

где

$$
g(\xi)=\frac{2 \pi N(1-\cos \xi)-3 \sin |\xi|+|\xi|(2+\cos \xi)}{\pi N \xi^{2}} .
$$

Для $\left|\omega_{0} t_{a}\right| \geq 2 \pi N$

$$
\frac{d W(\vartheta)}{d \Omega}=\frac{d W\left(\vartheta_{0}, 0\right)}{d \Omega} \frac{6}{\omega_{0}^{2} t_{a}^{2}} \frac{\sin ^{2} \vartheta}{\sin ^{2} \vartheta_{0}} .
$$

Для источника, размеры которого много больше характерной длины волны излучения $\lambda_{0}=2 \pi c / \omega_{0}$, основная часть энергии излучения сосредоточена в диапазоне углов $\vartheta$, таких, что $\left|\vartheta-\vartheta_{0}\right| \ll 1$ и ширина углового распределения определяется функцией $g(\xi)$. Значение $g(\xi)=1 / 2$ достигается при $\left|\xi_{1 / 2}\right| \approx 3.176$ для $N=1 \quad$ и $\left|\xi_{1 / 2}\right| \approx 2.783$ для $N \rightarrow \infty$. Соответственно ширина распределения энергии на полувысоте $\Delta \vartheta \approx \lambda_{0}\left|\xi_{1 / 2}\right| /\left(\pi a \cos \vartheta_{0}\right)$ для широкополосного излучения в $\approx 1.14$ раза больше, чем для узкополосного.

В работе [1] была приведена приближенная формула для угловой зависимости мощности излучения квадратной области размера $D$

$$
P\left(\theta_{x}, \theta_{y}\right)=P_{0}\left(\frac{\sin \psi_{x}}{\psi_{x}}\right)^{2}\left(\frac{\sin \psi_{y}}{\psi_{y}}\right)^{2},
$$

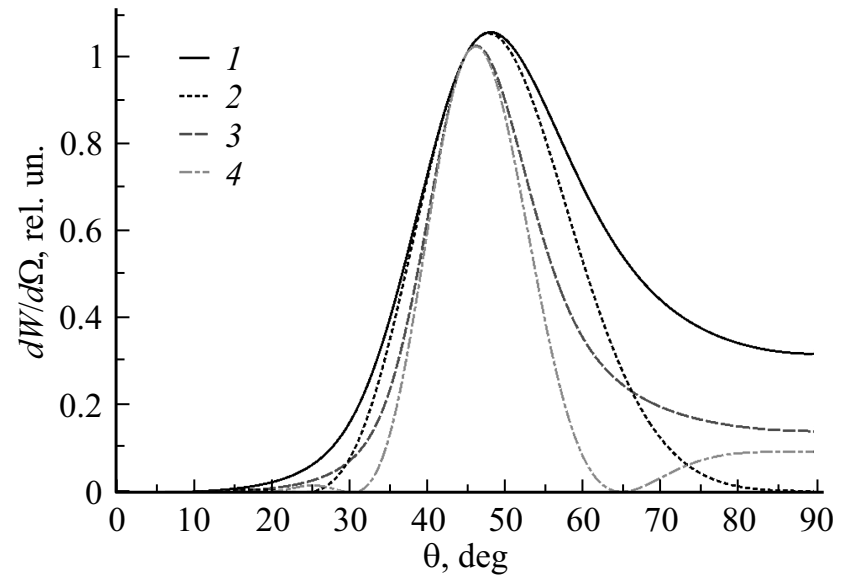

Рис. 2. Зависимость $d W(\vartheta) / d \Omega: 1-N=1, \omega_{0}=2 \pi \cdot 10 \mathrm{GHz}$; $2-N=100, \omega_{0}=2 \pi \cdot 10 \mathrm{GHz} ; 3-N=1, \omega_{0}=2 \pi \cdot 15 \mathrm{GHz} ;$ $4-N=100, \omega_{0}=2 \pi \cdot 15 \mathrm{GHz}$.

$$
\psi_{x}=\frac{\pi D \cos \vartheta_{0} \sin \theta_{x}}{\lambda}, \psi_{y}=\frac{\pi D \sin \theta_{y}}{\lambda}
$$

где $P_{0}$ - мощность излучения в направлении зеркального отражения $n_{1}, \lambda$ - характерная длина волны излучения, $\theta_{x}, \theta_{y}-$ угловое смещение от направления $n_{1}$ в плоскости $x-z$ и в плоскости $y-n_{1}$. Ширина распределения на полувысоте в плоскости $x-z\left(\psi_{y}=0\right)$ для случая $\left|\vartheta-\vartheta_{0}\right| \ll 1$ равна $\Delta \vartheta \approx 2.784 \lambda /\left(\pi D \cos \vartheta_{0}\right)$, что совпадает с полученным выше значением для узкополосного источника.

На рис. 2 показаны графики $(d W(\vartheta, 0) / d \Omega) /$ $\left(d W\left(\vartheta_{0}, 0\right) / d \Omega\right)$, полученные по формулам $(13),(14)$ для $\varphi=0, a=10 \mathrm{~cm}, \vartheta_{0}=45^{\circ}$, широкого и узкого спектра излучения и двух значений частот.

\section{Заключение}

Для прямоугольной области, электромагнитное излучение которой инициируется со сверхсветовой фазовой скоростью, в дипольном приближении найдено угловое распределение напряженности электрического поля в волновой зоне (4)-(7). Напряженность поля определяется поверхностной плотностью дипольного момента, которая может быть вычислена или измерена экспериментально, а также параметрами $t_{a}$ и $t_{b}$ (3), зависящими от размеров излучающей области и направления излучения.

Проведено спектральное разложение излучения (9), из которого следует, что наиболее высокие частоты излучаются в направлении зеркального отражения. При смещении от этого направления спектр сдвигается в область низких частот. Получено выражение для вычисления углового распределения энергии излучения (10). Проведены вычисления для случая, когда вторая производная дипольного момента от времени пропорциональна целому числу периодов синуса. 


\section{Список литературы}

[1] Carron N.J., Longmire C.L. // IEEE Transactions on Nuclear Science. 1976. Vol. NS-23. N 6. P. 1897-1902.

[2] Carron N.J., Longmire C.L. // IEEE Transactions on Nuclear Science. 1978. Vol. NS-25. N 6. P. 1329-1335.

[3] Лазарев Ю.Н., Петров П.В. // Письма в ЖЭТФ. 1994. Т. 60. Вып. 9. С. 625-628.

[4] Лазарев Ю.Н., Петров П.В. // ЖЭТФ. 1999. Т. 88. Вып. 5. C. $926-935$.

[5] Кондратьев А.А., Лазарев Ю.Н., Потапов А.В., Тищенко А.С., Заволоков Е.В., Сорокин И.А. // ДАН. 2011. Т. 438. Вып. 5. С. 615-618.

[6] Лазарев Ю.Н., Петров П.В., Сырцова Ю.Г. // Физика плазмы. 2003. Т. 29. Вып. 6. С. 527-539.

[7] Солдатов А.В.// Физика плазмы. 2005. Т. 31. Вып. 4. С. 336341.

[8] Ландау Л.Д., Лившиц Е.М. Теоретическая физика: Учебное пособие. В 10 т. Т. II. М.: Наука, 1988. 512 с. 